\title{
Influence of Lactobacillus kefiri on Intestinal Microbiota and Fecal IgA Content of Healthy Dogs
}

\author{
Alba Gaspardo ${ }^{1}$, Augusta Zannoni 1,2, Silvia Turroni ${ }^{3,4}$, Monica Barone ${ }^{3}$, \\ Maria Chiara Sabetti ${ }^{1}$, Renato Giulio Zanoni ${ }^{1}$, Monica Forni ${ }^{1,2}$, Patrizia Brigidi ${ }^{3,4}$ and \\ Marco Pietra ${ }^{1 *}$ \\ ${ }^{1}$ Department of Veterinary Medical Sciences, University of Bologna, Ozzano Dell'Emilia, Italy, ${ }^{2}$ Health Sciences and \\ Technologies - Interdepartmental Center for Industrial Research (CIRI-SDV), University of Bologna, Bologna, Italy, ${ }^{3}$ Unit of \\ Microbial Ecology of Health, Department of Pharmacy and Biotechnology, University of Bologna, Bologna, Italy, \\ ${ }^{4}$ Interdepartmental Centre for Agri-Food Industrial Research, University of Bologna, Bologna, Italy
}

\section{OPEN ACCESS}

Edited by:

Thomas Schermerhorn

Kansas State University, United States

Reviewed by:

Jan S. Suchodolski,

Texas A\&M University, United States

Silke Salavati,

University of Edinburgh,

United Kingdom

*Correspondence:

Marco Pietra

marco.pietra@unibo.it

Specialty section:

This article was submitted to

Comparative and Clinical Medicine,

a section of the journa

Frontiers in Veterinary Science

Received: 28 November 2019

Accepted: 27 February 2020

Published: 02 April 2020

Citation:

Gaspardo A, Zannoni A, Turroni S, Barone M, Sabetti MC, Zanoni RG,

Forni M, Brigidi P and Pietra M (2020)

Influence of Lactobacillus kefiri on Intestinal Microbiota and Fecal IgA

Content of Healthy Dogs.

Front. Vet. Sci. 7:146

doi: 10.3389/fvets.2020.00146
The increasing incidence of gastrointestinal tract pathologies in dogs and the worrisome topic of antibiotic resistance have raised the need to look for new therapeutic frontiers. Of these, the use of probiotics represents a potential therapeutic alternative. Lactobacillus kefiri $(L k)$ is a species of Lactobacillus isolated from kefir. Previous studies have demonstrated that its administration in mice downregulates the expression of proinflammatory mediators and increases anti-inflammatory molecules in the gut immune system. It also regulates intestinal homeostasis, incrementing immunoglobulin A (IgA) secretion. Since $L k$ has never been studied as a single probiotic in dogs, the aim of this study was to evaluate the safety of $L k$ in dogs, and its effect on IgA secretion and on intestinal microbiota composition. Ten healthy dogs without a history of gastrointestinal diseases were included. The dogs received $L k$ at a dose of $10^{7}$ live microorganisms orally, once daily for 30 days. The fecal samples were tested before administration, in the middle, at the end, and 30 days after discontinuation. The IgA secretion concentration and the microbiota composition were evaluated on the fecal samples. The results in this study suggested that $L k$ did not influence the concentration of IgA, nor significant changes of the intestinal microbiota were observed during and after the treatment. Therefore, additional studies are needed to investigate if a higher daily dosage of $L k$ can influence the intestinal homeostasis of dogs.

Keywords: dog, stool, gut microbiota, probiotic, IgA

\section{INTRODUCTION}

In recent years, intestinal microbiota has become increasingly relevant for veterinary scientists and has been studied for its role on the welfare of the host (1). It is an ecosystem including mainly bacteria, but also archaea, fungi, protozoa, and viruses, which plays several roles in the host physiology by means of a range of metabolic and immunological interactions. In fact, this complex ecosystem helps in the digestion of food by assisting the absorption and metabolism of nutrients, and has trophic and protective functions (2). It defends the gastrointestinal tract (GIT) against pathogenic organisms, promotes mucus production and enterocyte turnover, and modulates host immune development and functionality (3). In particular, commensal bacteria provide intestinal 
immune protection by regulating, among other things, the secretion of IgA, the lack of which seems to be correlated with chronic enteropathies in dogs $(4,5)$.

Canine chronic enteropathies, categorized into four classes (food responsive; antibiotic responsive; immunosuppressant responsive; nonresponsive enteropathy) according to the response to treatment, are multifactorial diseases where host genetic factors, the immune system, and indigenous intestinal bacteria are supposed to be engaged in intricate interactions $(6,7)$.

Dogs affected by antibiotic responsive enteropathy (ARE) are generally young, predominantly belong to large breeds, and show remission of clinical signs following antimicrobial administration (metronidazole, tylosin, doxycycline, rifaximine) $(8,9)$. It is thought that antimicrobials are able to modify the intestinal microbial population by counteracting its imbalances (i.e., dysbiosis); however, although a short-term response to metronidazole and tylosin has been reported, very few papers have described the long-term control of ARE (9-11).

Moreover, there is now evidence that prolonged treatment with antibiotics, particularly with metronidazole, can lead to permanent unfavorable changes in the microbiota, promoting antimicrobial resistance, currently one of the most important threats to public health $(12,13)$.

Therefore, the increasing incidence of GIT pathologies and the worrisome topic of antibiotic resistance create the need for new therapeutic options (14), and toward replacing antibiotics with (a) probiotics (live microorganisms that confer a healthy benefit on the host); (b) prebiotics (a substrate selectively utilized by host microorganisms useful for reestablishing a eubiotic microbiota layout); (c) synbiotics (a mixture of probiotics and prebiotics having a synergistic action on host health); and (d) postbiotics (soluble factors or metabolic byproducts, secreted by live bacteria or released after bacterial lysis expressing biological activity in the host).

Lactic acid bacteria (LAB), including Lactococcus, Streptococcus, Enterococcus, Pediococcus, Leuconostoc, and Lactobacillus, are probiotic bacteria that are normally part of the intestinal microbiota of dogs and cats (2). Lactobacillus species are distributed throughout the canine intestinal tract in varying amounts (2). Several strains of Lactobacilli have specifically been studied for their ability to reduce the number of pathogenic bacteria in the canine intestine (15-20).

Among different naturally fermented foods and their potential probiotic properties, particular attention has recently been focused on kefir, a dairy product that could modulate canine intestinal microbiota if given regularly (21). Kefir has a complex composition of microbial organisms, which includes several species of LAB as well as acetic acid bacteria and yeast (22). Lactobacillus kefiri ( $L k)$ is a Lactobacillus species that has been isolated from kefir (23). Previous studies have demonstrated that its administration in mice downregulates the expression of proinflammatory mediators and increases anti-inflammatory molecules in the gut immune system $(24,25)$. It also regulates intestinal homeostasis by increasing IgA secretion and mucin production (25). Probiotic properties of $L k$ have also recently been demonstrated in humans (26); however, to the best of the authors' knowledge, the use of $L k$ has never been evaluated in dogs.

The aim of this study was to evaluate the safety and ease of administration of $L k$ in healthy dogs and its ability to impact the intestinal microbiota composition and IgA secretion.

\section{MATERIALS AND METHODS}

\section{Lactobacillus kefiri Administration}

This study is based on the use of a commercial food supplement (Kefibios ${ }^{\circledR}$ ), provided by the company Hulka S.r.L. (Rovigo, Italy), containing live lactic ferments of $L k$ (LKF01-DSM 32079), currently used as probiotics in human medicine. Copyright permission to publish the product name (Kefibios ${ }^{\circledR}$ ) was given by the Chief Executive Officer of Hulka S.r.L. The product is marketed in capsules. Following the label of the product, five drops of the solution reconstituted with $6 \mathrm{ml}$ of vegetable oil in prefilled bottles contain $\geq 10^{9}$ active fluorescent units (AFUs) of live and viable $L k$ (ISO 19344:2015). The dose corresponds to the human one indicated by the company, regardless of age and body weight (BW). The study, involving 10 healthy privately owned dogs, was conducted at the Veterinary Teaching Hospital (VTH) of our department. The owners were carefully instructed as to how to use and mix the product, and then shake the bottle before each administration. The product was then stored at room temperature between $10^{\circ} \mathrm{C}$ and $25^{\circ} \mathrm{C}$ and away from direct light.

The recruitment of the dogs in the study was voluntary and at no cost to the owners. Written informed consent before enrollment in the study was obtained from the owners.

The trial was authorized by the Animal Welfare Committee of the University of Bologna (Protocol No. 3885 of 21/07/2017).

\section{Animals and Experimental Design}

Privately owned dogs of various breeds, genders, and weight, over 1 year of age, were enrolled in the trial. Dogs with any disease in the previous 2 months before the start of the trial were excluded. The inclusion criterion was the absence of antimicrobial or immunosuppressive treatment up to 60 days prior to the start of and during the trial. For inclusion, each dog was evaluated with a clinical examination and a laboratory panel, which included a complete blood count, a serum biochemistry profile, and coprological examination for gastrointestinal parasites.

The body condition score (BCS) was calculated using the 19 score proposed by Royal Canine SAS, and the fecal score was evaluated according to the Fecal Score System (FSS 1-7) proposed by Nestlé-Purina Petcare. The BCS was determined as follows: $1-3=$ too thin, $4-5=$ ideal, and $6-9=$ too heavy. The fecal samples were scored as follows: $1=$ very hard and dry, leaves no residue on the ground when picked up; $2=$ firm, but not hard, pliable, little or no residue on the ground when picked up; $3=$ log-shaped, moist surface, leaves residue on the ground, but holds form when picked up; $4=$ very moist and soggy, leaves residue on the ground and loses form when picked up; $5=$ very moist but has a distinct shape, leaves residue on the ground and loses form when picked up; $6=$ has texture, but no defined shape, leaves residue on the ground when picked up; and $7=$ watery, no texture, present in flat patches. 
TABLE 1 | Dogs included in the study.

\begin{tabular}{|c|c|c|c|c|c|c|c|}
\hline Dogs & Breed & Gender & Age & Weight & BCS & Fecal score & Diet \\
\hline 1 & Mix breed & C & $4 \mathrm{y} 1 \mathrm{~m}$ & $33 \mathrm{~kg}$ & 6 & 2 & Purina tonus dog chow chicken \\
\hline 2 & Mix breed & S & 4yOm & $18.3 \mathrm{~kg}$ & 6 & 2 & Purina tonus dog chow chicken \\
\hline 3 & Border Collie & $\mathrm{F}$ & $2 \mathrm{y} 6 \mathrm{~m}$ & $19 \mathrm{~kg}$ & 4 & 2 & Prolife adult medium chicken and rice \\
\hline 4 & Australian shepherd & S & 6yOm & $23 \mathrm{~kg}$ & 5 & 2 & Royal canin veterinary diet neutered adult medium dog \\
\hline 5 & Border Collie & C & $2 \mathrm{y} 6 \mathrm{~m}$ & $20.5 \mathrm{~kg}$ & 5 & 2 & Farmina ancestral low grain lamb and blueberry \\
\hline 6 & Dachshund & $\mathrm{F}$ & $3 y 4 m$ & $5.6 \mathrm{~kg}$ & 6 & 2 & Royal canin small dog chicken and rice \\
\hline 7 & Border Collie & S & $9 y 7 m$ & $19 \mathrm{~kg}$ & 5 & 2 & Prolife adult medium chicken and rice \\
\hline 8 & Labrador retriever & M & $2 \mathrm{y} 5 \mathrm{~m}$ & $32.1 \mathrm{~kg}$ & 5 & 3 & Monge natural superpremium rabbit, rice, and potatoes \\
\hline 9 & Mix breed & S & $9 y 8 m$ & $7.5 \mathrm{~kg}$ & 5 & 3 & Royal canin small dog chicken and rice \\
\hline 10 & Mix breed & $\mathrm{C}$ & $5 y 11 \mathrm{~m}$ & $5.7 \mathrm{~kg}$ & 5 & 2 & Royal canin small dog chicken and rice \\
\hline
\end{tabular}

M, male; $C$, neutered male; F, female; $S$, neutered female; BCS, body condition score.

Not being experimental animals but privately owned dogs, diet was not standardized during the trial but the previous diet (Table 1) was maintained, and water was supplied ad libitum.

During the entire experimental time, the dogs received five drops ( $\geq 10^{9}$ AFUs) of $L k$ once daily for 30 days, administered directly in the mouth during the dinner meal.

Clinical examination, BCS, and FSS were performed at inclusion, and, during the trial, respectively, 15, 30, and 60 days after the start of $L k$ administration.

Samples of fresh feces were collected from each dog on three consecutive days: (a) at T0, before the start of $L k$ administration (days $-3,-2,-1$ ); (b) at T15, in the intermediate time of $L k$ administration (days 13, 14, 15); (c) at T30, at the end of $L k$ administration (days 28, 29, 30); (d) at T60, 1 month after the last $L k$ administration (days $58,59,60$ ).

The samples were collected by the owner immediately after defecation, immediately stored at $-20^{\circ} \mathrm{C}$ in the owner's household freezer until delivered frozen to the department where they were stored at $-80^{\circ} \mathrm{C}$ until use.

All the samples were analyzed for IgA detection, while, for the gut microbiota, a pool of three samples from each dog at T0, T30, and T60 was prepared and analyzed.

\section{Fecal Microbiota Analysis}

Microbial DNA was extracted from about $250 \mathrm{mg}$ of pooled fecal sample, derived from the experimental points (T0; T30; T60) for each dog, using the repeated bead-beating plus column method, as previously described (27). Briefly, samples were suspended in $1 \mathrm{ml}$ of lysis buffer $(500 \mathrm{mM} \mathrm{NaCl}, 50 \mathrm{mM}$ Tris- $\mathrm{HCl}, \mathrm{pH} 8$, $50 \mathrm{mM}$ EDTA, and 4\% SDS) and bead-beaten three times in a FastPrep instrument (MP Biomedicals, Irvine, CA, USA) at 5.5 movements/s for $1 \mathrm{~min}$, in the presence of four 3-mm glass beads and $0.5 \mathrm{~g}$ of $0.1-\mathrm{mm}$ zirconia beads (BioSpec Products, Bartlesville, OK, USA). After incubation at $95^{\circ} \mathrm{C}$ for $15 \mathrm{~min}$, samples were centrifuged at 13,000 $\mathrm{rpm}$ for $5 \mathrm{~min}$. Two hundred and sixty microliters of $10 \mathrm{M}$ ammonium acetate was added to the supernatant, followed by 5-min incubation on ice and 10min centrifugation at $13,000 \mathrm{rpm}$. The supernatant was added with one volume of isopropanol, followed by incubation on ice for $30 \mathrm{~min}$. Precipitated nucleic acids were washed with $70 \%$ ethanol, resuspended in $100 \mu \mathrm{l}$ of TE buffer, and treated with $2 \mu \mathrm{l}$ of $10 \mathrm{mg} / \mathrm{ml}$ DNase-free RNase at $37^{\circ} \mathrm{C}$ for $15 \mathrm{~min}$. DNA was further purified using the QIAamp Mini Spin columns (QIAGEN, Hilden, Germany) following the manufacturer's instructions. DNA concentration and quality were evaluated using the NanoDrop ND-1000 spectrophotometer (NanoDrop Technologies, Wilmington, DE, USA).

The V3-V4 hypervariable region of the 16S rRNA gene was amplified by using the $341 \mathrm{~F}$ and $785 \mathrm{R}$ primers with Illumina adapter overhang sequences, as previously described (28). PCRs were performed in a final volume of $25 \mu \mathrm{l}$, containing $12.5 \mathrm{ng}$ of genomic DNA, $200 \mathrm{nM}$ of each primer, and 2X KAPA HiFi HotStart ReadyMix (Kapa Biosystems, Wilmington, MA, USA), in a Thermal Cycler T (Biometra GmbH, Göttingen, DE) with the following thermal cycle: initial denaturation at $95^{\circ} \mathrm{C}$ for $3 \mathrm{~min}$, 25 cycles of denaturation at $95^{\circ} \mathrm{C}$ for $30 \mathrm{~s}$, annealing at $55^{\circ} \mathrm{C}$ for $30 \mathrm{~s}$ and extension at $72^{\circ} \mathrm{C}$ for $30 \mathrm{~s}$, and a final extension step at $72^{\circ} \mathrm{C}$ for $5 \mathrm{~min}$. Amplicons were purified using a magnetic bead-based system (Agencourt AMPure XP; Beckman Coulter, Brea, CA, USA). Indexed libraries were prepared by limited-cycle PCR using Nextera technology and further purified as described above. Final libraries were pooled at equimolar concentration, denatured with $0.2 \mathrm{~N} \mathrm{NaOH}$, and diluted to $6 \mathrm{pM}$ before loading onto the MiSeq flow cell. Sequencing was performed on Illumina MiSeq platform with a $2 \times 250$ bp paired-end protocol, according to the manufacturer's instructions (Illumina, San Diego, CA, USA).

Sequencing Reads Were Deposited in the National Center for Biotechnology Information Sequence Read Archive (NCBI SRA; BioProject ID PRJNA 592436).

\section{Quantification of IgA Fecal Content}

Stool samples were thawed and subsequently freeze-dried (Modulyo EF4, 1044, Edwards, Apeldoorn, The Netherlands) for $16 \mathrm{~h}$ in order to eliminate the water contained and standardize the subsequent analysis, as reported by Grellet et al. (29). The lyophilized fecal samples were resuspended in 1X PBS (phosphate buffer saline) containing 0.5\% Tween20 (SigmaAldrich, St. Louis, MO, USA), according to a weight/volume ratio of $100 \mathrm{mg} / \mathrm{ml} \mathrm{(1:10} \mathrm{dilution)} \mathrm{by} \mathrm{vortex} \mathrm{(3')} \mathrm{and} \mathrm{then}$ 
centrifuged at $1,500 \times g$ for $10 \mathrm{~min}$. After removing the supernatant, a further centrifugation was carried out at 10,000 $\times g$ for $20 \mathrm{~min}$, then the aqueous phase was taken and frozen at $-20^{\circ} \mathrm{C}$ until the processing moment. The determination of the IgA secretion amount was carried out by using a commercial kit (dog IgA ELISA Quantitation Set, Bethyl Laboratories Inc., TX, USA; Assay Range: 15.6-1,000 ng/ml), following the manufacturer's protocol.

\section{Kefibios ${ }^{\circledR}$ Quality Control}

In order to confirm the content of $L k$ declared by the manufacturer, we performed an independent assessment of $L k$ concentration in Kefibios ${ }^{\circledR}$ capsules (Bacteriological Laboratory, UNI EN ISO 9001:2015 registration number IT-15164).

Five different batches of the product, acquired in several drugstores, with expiration date from 1 to 2 years with respect to the time of the analysis, were analyzed, and enumeration of viable bacteria was conducted by the plate count method.

Briefly, each sample was firstly solubilized in MRS Broth (Oxoid CM359, Thermo Scientific, MA, USA) and gently shaken at room temperature for $15 \mathrm{~min}$ by using an orbital shaker, then serially diluted in the same broth and inoculated onto MRS Agar (Oxoid CM361, Thermo Scientific, MA, USA) plates. Plating was performed in triplicate.

Plates were incubated at $37^{\circ} \mathrm{C}$ for $72 \mathrm{~h}$ under anaerobic atmosphere, and the number of colony-forming units (CFUs) was determined.

The colonies obtained in the tests were identified by the API $50 \mathrm{CHL}$ (BioMerieux, FI, Italy) test, and results are presented as the number of viable cells per capsule and per dose (five drops).

\section{Bioinformatics and Statistics}

For the analysis of fecal microbiota composition and diversity, raw sequences were processed using a pipeline combining PANDAseq (30) and QIIME 1 (31). High-quality reads were binned into operational taxonomic units (OTUs) through an open-reference strategy at 0.97 similarity threshold by using UCLUST (32). Taxonomy was assigned using the RDP classifier and Greengenes as a reference database (release May 2013). All singleton OTUs and chimeras, identified by ChimeraSlayer (33), were discarded. Alpha rarefaction was performed using observed OTUs, Shannon, and the phylogenetic diversity (PD) whole tree metrics, while beta diversity was estimated by computing weighted and unweighted UniFrac distances. For the identification of $L k$, OTUs assigned to the genus Lactobacillus were subjected to BLAST analysis (34).

All statistical analyses were performed in $\mathrm{R}$ (version 3.1.3) using the packages vegan, made4, and GraphPad Prism V.5.01 (GraphPad Software, La Jolla, CA, USA).

Assessment of data for normality was carried out by applying the D'Agostino and Pearson Omnibus normality test.

UniFrac distances were used for principal coordinates analysis (PCoA), and the significance of data separation was tested using a permutation test with pseudo-F ratios (function Adonis of vegan). Wilcoxon test for paired data was used to assess significant differences in alpha diversity and taxon relative abundance between groups.
A repeated-measures ANOVA (with Tukey post hoc test) was applied to evaluate the differences in BW and fecal IgA content between experimental points (T0, T15, T30, and T60). Friedman test (with Dunn's as post hoc test) was applied to evaluate the differences in BCS and FSS between experimental points (T0, $\mathrm{T} 15$, T30, and T60).

$p<0.05$ was considered statistically significant.

\section{RESULTS}

\section{Animals}

Ten healthy adult dogs were included. Of those, one was male, three neutered males, two females, and four neutered females. Mean age was $4.9 \pm 2.8$ years (range $2-9)$. Breeds included were mixed breed dogs $(n=4)$, Border Collies $(n=3)$, Labrador Retriever $(n=1)$, Australian Shepherd $(n=1)$, and Dachshund $(n=1)$. Their BW ranged from 5 to $33 \mathrm{~kg}(18.3 \pm 9.84 \mathrm{~kg})$, while their average BCS was normal (range 4-6). Initial FSS was normal in all dogs (range 2-3; Table 1). All dogs had normal hematological and biochemical parameters, and the coprological examination was negative for parasites.

The liquid product containing $L k$ was spontaneously accepted by all the subjects. No clinical signs during the trial (with the exception of $\operatorname{dog} \# 6$ ), and up to 1 month later, were reported by the owners.

There were no significant changes in BW and BCS during the study period, nor did the FSS of each animal changed.

Dog \#6 developed a urinary tract infection at day 50 of the trial that required antibiotic treatment. This dog's last fecal sample was therefore excluded from the analysis.

\section{Microbiota Analysis}

A total of $1,536,903$ high-quality reads (mean $\pm \mathrm{SD}, 52,997$ $\pm 16,302$ ) were obtained and clustered into 2,716 OTUs at $97 \%$ similarity.

The PCoA of intersample diversity based on weighted and unweighted UniFrac distances showed no significant separation among the study groups (i.e., baseline, end of treatment, and follow-up; $P=0.7$, permutation test with pseudo-F ratios; Figure 1A). Similarly, no significant differences were found in alpha diversity after $L k$ administration or in the follow-up compared to the baseline ( $P>0.05$, Wilcoxon test; Figure 1B).

In line with the available literature on the gut microbiota of healthy dogs $(35,36)$, the phylum-level microbial profiles at the baseline were dominated by Firmicutes (relative abundance, mean \pm SEM, $73.1 \pm 4.2 \%$ ), with Actinobacteria (14.2 \pm $3.1 \%)$, Bacteroidetes (7.8 $\pm 2.2 \%)$, and Fusobacteria (3.9 $\pm 1.2 \%$ ) as minor components (Figure 2). Lachnospiraceae, Coriobacteriaceae, Clostridiaceae, and Erysipelotrichaceae were the major families of the baseline microbiota (relative abundance $\geq 10 \%$; Figure 3A). Consistently, the most represented genera were Blautia, Clostridium, and Collinsella (Figure 3B). Following $L k$ administration, no significant differences in taxon relative abundance at these phylogenetic levels (i.e., phylum, family, and 

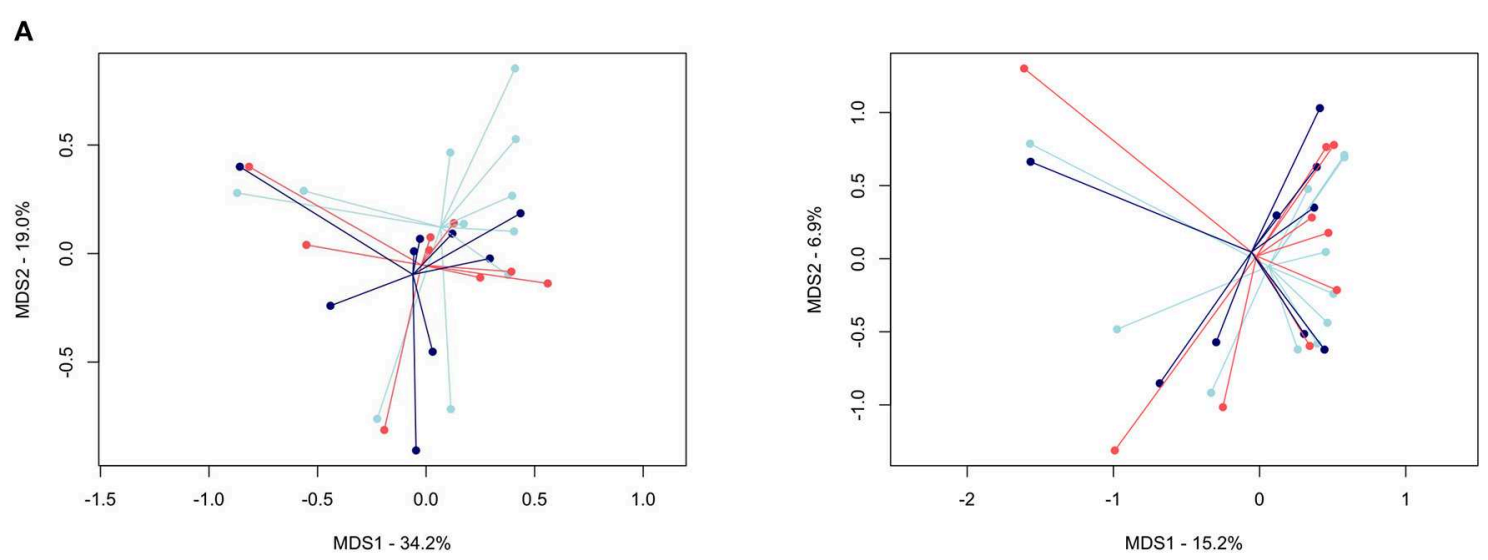

B

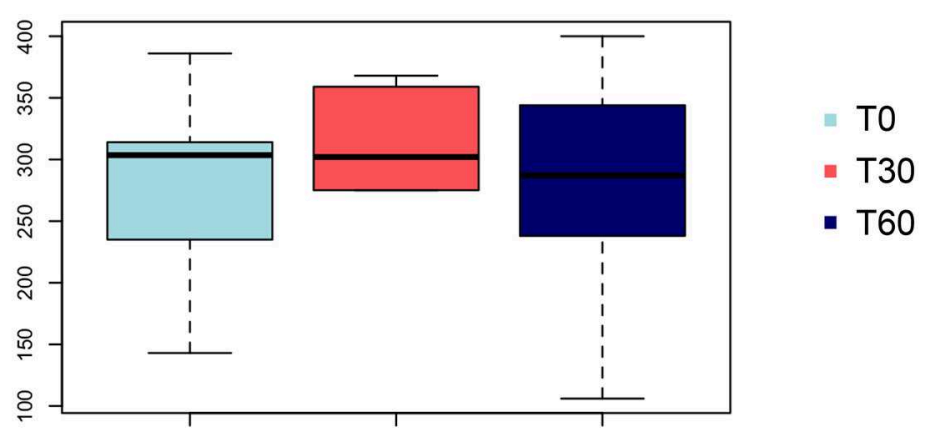

FIGURE 1 | Gut microbiota diversity of healthy dogs following $L k$ administration. (A) Principal coordinates analysis of intersample diversity, based on weighted (left) and unweighted (right) UniFrac distances. (B) Alpha diversity computed with observed OTU metrics. T0, baseline; T30, after 30 days of $L k$ administration; T60, 1 month after the end of the treatment.

genus) were observed (Figures 2, 3). One month after the end of the treatment, these changes were no longer detectable.

However, at T60, a decreasing trend in the relative abundances of Fusobacteriaceae and Ruminococcaceae was found (T0 vs. T60, $P=0.11$ and 0.15 , respectively; Figure 3A). With specific regard to $L k$, OTUs assigned to this species were not present at T0 but accounted for $10.3 \%$ of Lactobacillus diversity (and $0.07 \%$ of the intestinal ecosystem) after 30 days of $L k$ administration, which then disappear again in the follow-up.

\section{IgA Content in Fecal Samples}

A total of 117 fecal samples were taken and analyzed. Three samples (T58, T59, and T60 from dog \#6) were missing because dog \#6 was excluded from the trial.

The water content of feces was very similar among all the samples with percentage values of $38.08 \pm 4.97$ (mean \pm SD).

IgA was detected in all samples except for sample T15 from $\operatorname{dog} \# 3$ (undetectable $<15.6 \mathrm{ng} / \mathrm{ml}$ ). All dogs have shown a huge intra-individual variability of fecal IgA content among the three following day samples at T0, T15, T30, and T60 (Figure 4A).

Only in four dogs it was possible to observe an increasing trend in the IgA fecal content (dogs 1, 2, 3, and 6; Figure 4A).

In Figure 4B, we showed a ratio between the quantity of IgA fecal content at T15, T30, and T60 and the basal (T0) for each dog.
Overall, the fecal IgA content between different time points did not show any significant differences ( $p=0.1$; Figure 5).

\section{Kefibios ${ }^{\circledR}$ Quality Control}

The number of viable $L k$ per capsule from different product batches varied from $7.73 \times 10^{7} \mathrm{CFU}$ to $152 \times 10^{7} \mathrm{CFU}(81 \pm$ $61 \times 10^{7} \mathrm{CFU}$, mean and standard deviation).

The number of viable $L k$ in the liquid formulation per dose of five drops varied from $0.39 \times 10^{7} \mathrm{CFU}$ to $7.55 \times 10^{7} \mathrm{CFU}$ $\left(3.2 \pm 2.4 \times 10^{7} \mathrm{CFU}\right.$, mean and standard deviation) and was hence 2-log fold lower than the declared concentration $\left(\geq 10^{9}\right.$ $\mathrm{CFU})$ by the company.

\section{DISCUSSION}

In recent years, interest in characterizing the canine intestinal microbiota has soared, and therapeutical interventions that can positively influence the microbiota composition and function, specifically identification of novel probiotics, are sought $(2,3,37)$.

Several potential probiotics have already been tested in dogs, including bacterial species belonging to the genera Enterococcus, Lactobacillus, Bifidobacterium, and Saccharomyces, demonstrating their role in the treatment of acute and chronic enteropathies (15-18, 38-40). 


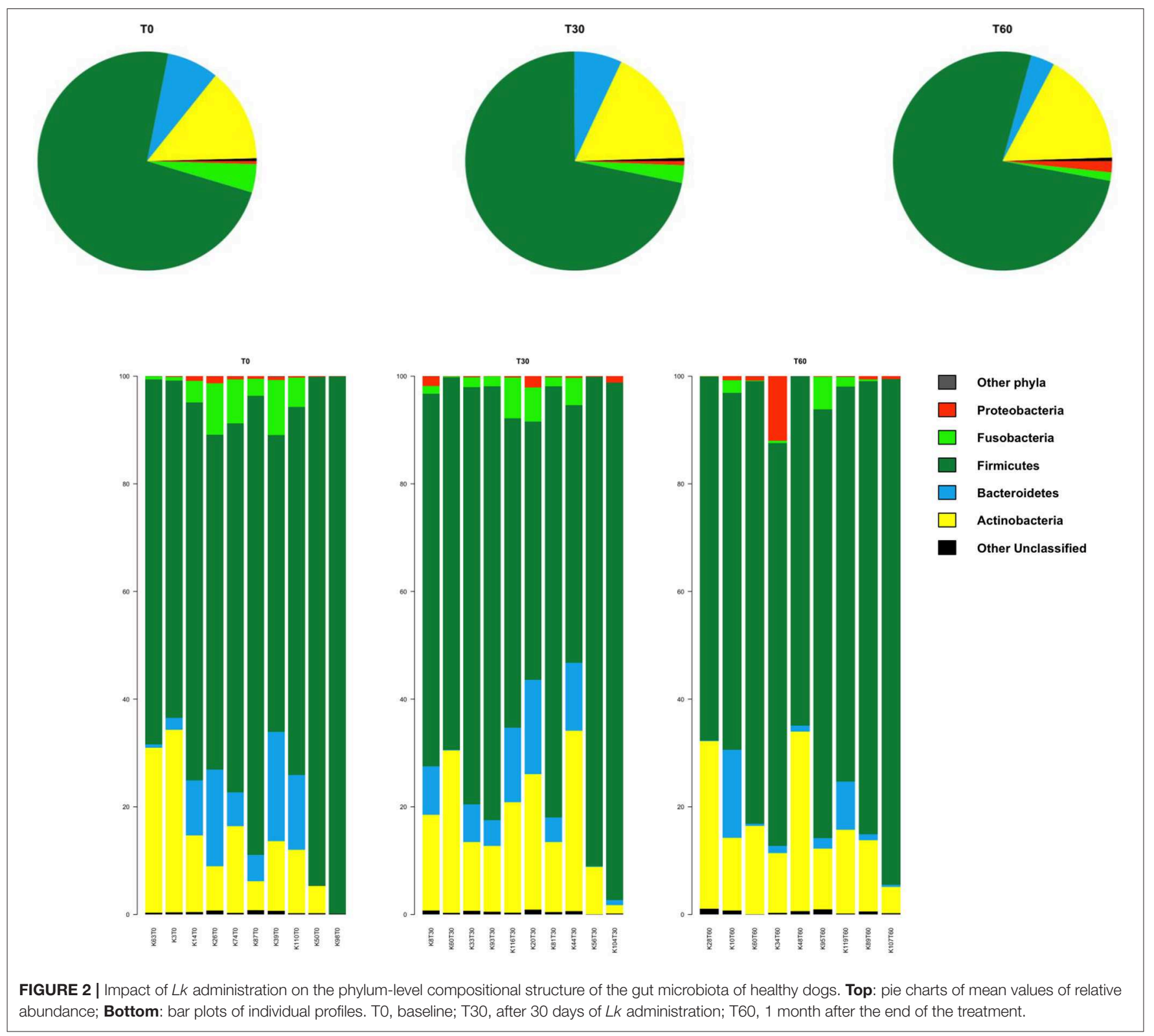

Among Lactobacillus strain, several species have been already studied (Lactobacillus acidophilus DSM13241; Lactobacillus fermentum CCM7421; Lactobacillus animalis LA4). However, the achieved results are difficult to compare as different dosages $\left(10^{7}\right.$ $\mathrm{CFU} /$ daily dose; $10^{9} \mathrm{CFU} /$ daily dose; 3 to $3.6 \times 10^{9} \mathrm{CFU} /$ daily dose) and different forms of application were used, and the duration of administration was also variable $(15,17,18)$.

To date, no studies have evaluated the influence of $L k$ on the parameters of intestinal health in dogs.

Only one previous study has assessed the effect of kefir (a fermented dairy product containing $L k$ ) on dogs (21). This study demonstrated a significant increase in the fecal $\mathrm{LAB}$ :Enterobacteriaceae ratio and a decrease in the fecal Firmicutes:Bacteroidetes ratio, which was interpreted as an improvement of the gut microbiota composition. However, this study did not use a single bacterial strain, but the mixture of more than 50 microorganisms contained in kefir, and only reported a dose for total LAB $\left(9.32 \pm 0.23 \log _{10} \mathrm{CFU} / \mathrm{ml}\right)$ and yeast $(7.12 \pm$ $\left.0.36 \log _{10} \mathrm{CFU} / \mathrm{ml}\right)(21)$. It is therefore difficult to calculate and compare the precise concentration of $L k$ that was administered in this study, and to infer the changes observed to a single potentially probiotic strain, as it could be attributed to several microorganisms and their potential synergism.

In addition, the composition of microorganisms in kefir may vary depending on its origin, the substrate used in the fermentation process, and the culture maintenance methods (41).

The product used in our study is a commercial preparation registered as a probiotic for human medicine, containing $L k$ with 30-day stability of reconstituted product guaranteed by the producer. Currently, there are no recommendations for the 
A
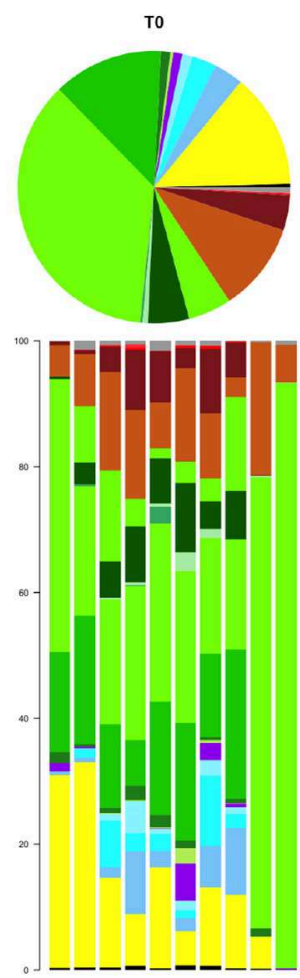

B
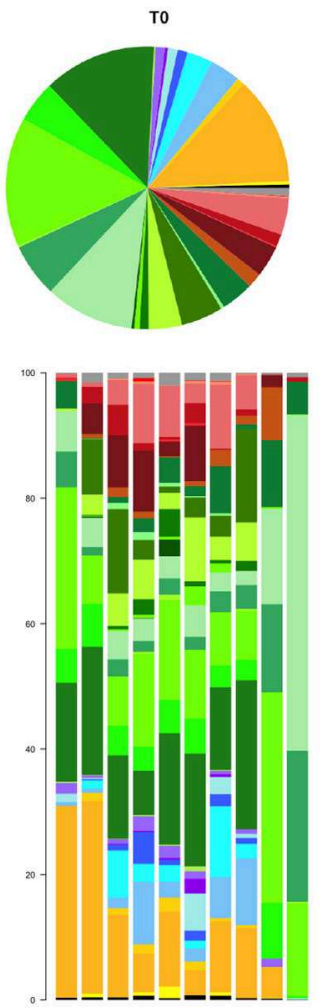
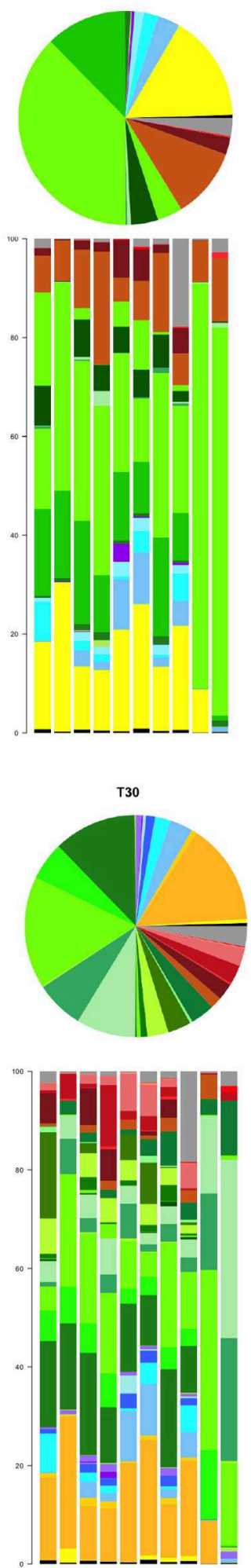

T60
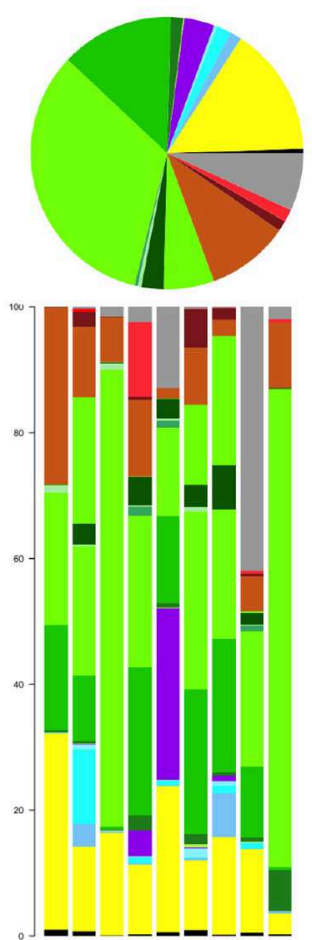

$\square \quad$ Other families

$\square$ Enterobacteriaceae

- Alcaligenaceae

- Fusobacteriaceae

$\square$ Erysipelotrichaceae

$\square \quad$ Veillonellaceae

- Ruminococcace

$\square \quad$ Peptostreptococcacea

$\square$ Peptococcaceae

$\square \quad$ Lachnospiraceae

Clostridiaceae

$\square$ Clostridiales Unclassifie

$\square \quad$ Turicibacteraceae

- Streptococcaceae

$\square \quad$ [Paraprevotellaceae]

$\square \quad$ Prevotellaceae

Bacteroidacea

Coriobacteriaceae

Other Unclassified
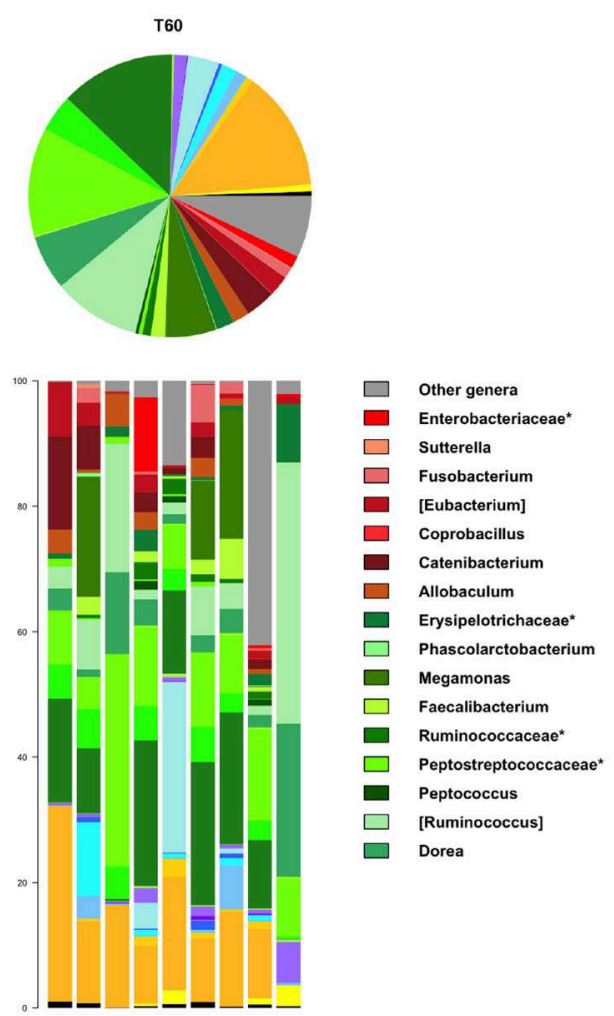

$\square$ Coprococcus

$\square \quad$ Blautia

$\square$ Lachnospiraceae $^{*}$

$\square$ Clostridium

$\square$ Clostridiaceae

$\square$ Clostridiales*

$\square$ Turicibacter

$\square$ Streptococcus

$\square \quad$ [Prevotella]

$\square$ Prevotella

$\square$ Bacteroides

$\square \quad$ Slackia

$\square$ Collinsella

$\square$ Coriobacteriaceae

- Other Unclassified

Ruminococcus]

FIGURE 3 | Impact of $L k$ administration on the family- and genus-level compositional structure of the gut microbiota of healthy dogs. Relative abundance profiles at family (A) and genus (B) level. Top: pie charts of mean values; bottom: bar plots of individual profiles. T0, baseline; T30, after 30 days of $L k$ administration; T60, 1 month after the end of the treatment. 


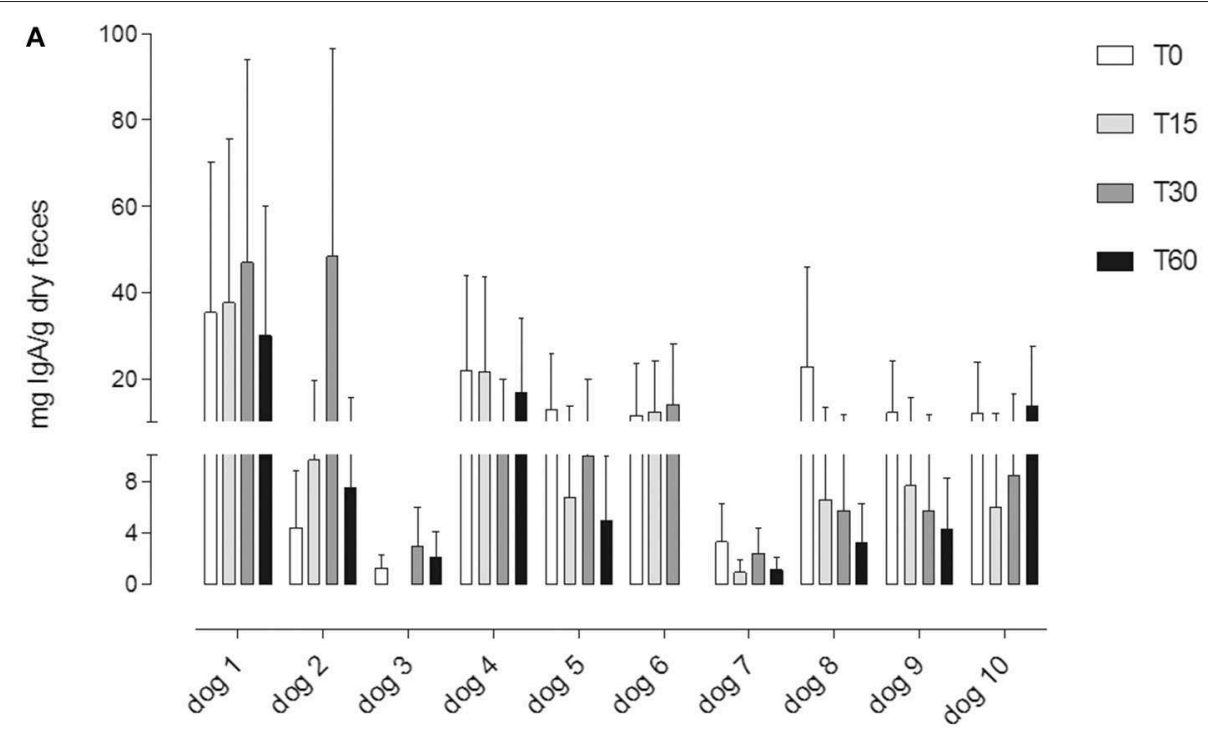

B

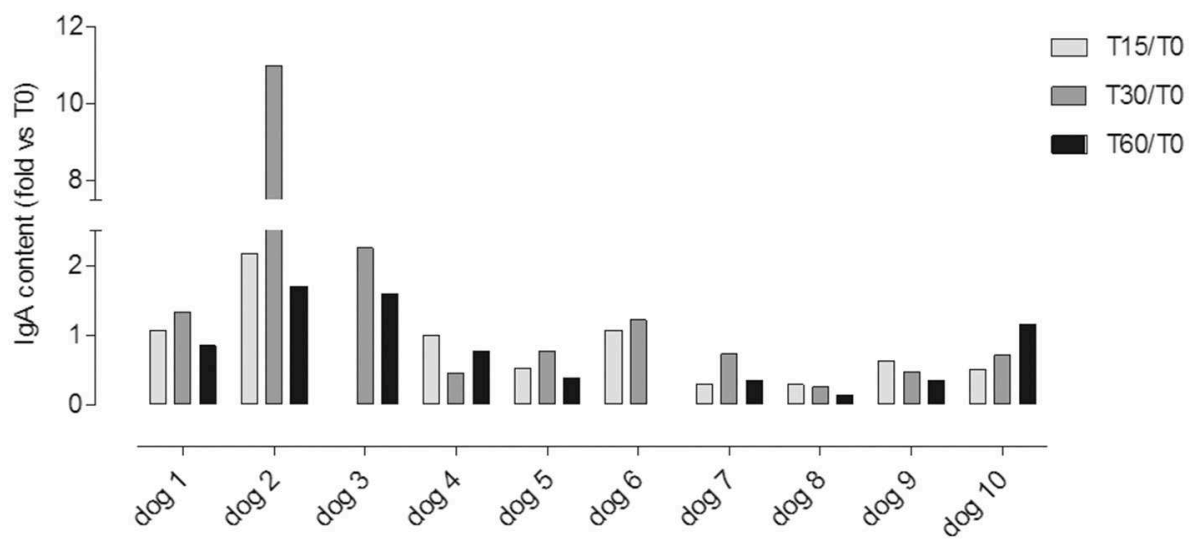

FIGURE 4 | (A) Fecal IgA content at different time points (T0; T15; T30; T60); each value (mean \pm SD) represents the average of the measurements of the three samples collected in three consecutive days for each experimental point: TO, before the administration, T15 and T30, after 15 and 30 days of $L k$ administration; and T60, 1 month after the last $L k$ administration. The T60 fecal samples of dog \#6 were excluded from the analysis due to antibiotic therapy. (B) IgA fecal content evaluated by the ratio between the quantity at T15, T30, and T60 and the basal (TO) for each dog.

dosage of $L k$ in dogs. Daily dosages used in mice and people were $10^{8}$ and $10^{10} \mathrm{CFU}$, respectively $(24,26)$.

The dose administered to dogs in the present study was extrapolated from the dose for an adult person as recommended by the manufacturer. While the daily dose for people (five drops) should contain $\geq 10^{9}$ AFUs of live and viable $L k$, the results presented here indicate that the same dose, administered to the dogs of this study, was more equal to $3.2 \pm 2.4 \times 10^{7} \mathrm{CFU}$.

The 30-day duration of the experiment was chosen based on available literature and a suspected washout time of 4 weeks after discontinuation of administration (42).

Fecal samples were chosen over other types of samples to evaluate the intestinal microbiota, as they can be collected in a noninvasive manner, raising no ethical concerns in comparison to, for example, intestinal mucosal biopsies. In addition, the ability of $L k$ to modulate microbiota composition has already been demonstrated using fecal samples in people and mice (25, 26). Similarly, concentrations of IgA from duodenal biopsies and fecal samples showed no difference in a previous study (4).

As for the gut microbiota, in line with what has already been reported for probiotic supplementations in healthy individuals (43), no changes were detected after $L k$ administration.

The IgA results showed a large interdog variability at T0 (from 1.33 to $35.35 \mathrm{mg} / \mathrm{g}$ of dehydrated feces), which, although all dogs appeared clinically healthy, could depend on the extreme variability in experimental dogs' signaling, age, life environment, and food taken.

Moreover, our IgA variability is in agreement with the data reported by other authors $(29,44)$.

Regardless of the basal value, we have noted the absence of a significant variation in the fecal IgA content comparing the experimental time points, i.e., pretreatment 


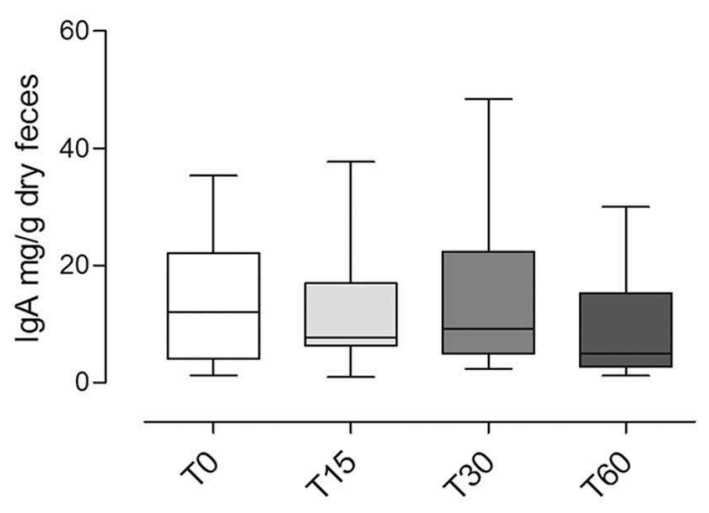

FIGURE 5 | Fecal IgA content at different time points (T0; T15; T30; T60) in dogs included in the trial (mean $\pm \mathrm{SD}$ ). No significant statistical differences were observed $(p=0.1)$.

(T0), during (T15 and T30), and 30 days after $L k$ administration (T60).

Possible explanation of the results could be related to a poor immunomodulatory effect of $L k$ toward the canine GALT (gut associated lymphoid tissue) due to (a) poor $L K$ viability in pharmaceuticals administered and consequent insufficient probiotic dose; (b) inadequate administration period; and (c) poor vitality in the gastrointestinal tract of the dog with lack of $L k$ probiotic activity in dogs.

Considering the first point, the differences found between the concentration declared by the company $\left(L k \geq 25 \times 10^{9}\right.$ AFU/capsule; $\geq 10^{9}$ AFU/dose) and that found in our quality control analysis $\left(81 \pm 61 \times 10^{7} \mathrm{CFU} /\right.$ capsule; $3.2 \pm 2.4 \times 10^{7}$ CFU/dose) must be emphasized.

It must be stressed that the gap between what was declared by the probiotic company and what was highlighted by an independent analysis is not a rare event. In fact, analyzing the literature, numerous papers, both in the field of human and veterinary medicine, evidence this gap, with several-folds reduction of live probiotics concentration with respect to which reported by the companies (45-47).

Though not much is known about the minimal dose and/or frequency of probiotics required for the probiotic effect, it seems to be dose-dependent (48). For this reason, it cannot be excluded that the absence of changes observed in fecal microbiota and IgA during the trial with $L k$ can be attributed to an insufficient dosage, corresponding to $\sim 3 \%$ of the expected dose indicated for humans.

It is also true that an improvement in the enteric immune function in dogs was observed even after the administration of Lactobacillus fermentum at the dosage of $1 \times 10^{7} \mathrm{CFU} /$ daily dose for 1 week, similar dose, and lower trial time, than the one actually used by us (18).

With respect to the treatment time, our study treatment is longer than employed in similar studies (15-17), with two intratreatment withdrawals in addition to pre- and posttreatment sampling, so this point can be excluded as a cause of poor response too.

Lastly, no previous studies have analyzed the $L k$ vitality in the canine gastrointestinal tract; therefore, it cannot be excluded that this may be the cause of the poor probiotic activity observed in our study.

Conversely, studies performed in human medicine have verified the gastrointestinal $L k$ vitality showing a high rate of adhesion of $L k$ to intestinal cells and strong resistance to gastric juice and intestinal bile salts (49).

Limitations of the study include the fact that dogs did not receive a uniform diet during the experiment, and the relatively low number of dogs included, further confounded by the exclusion of dog $\# 6$ from the analysis.

However, we believe that not artificially standardizing the dogs' diet might allow for results to be translated easier into reallife veterinary practice conditions and would showcase that $L k$ is able to impact the microbiota independent of the diet given. We also believe that our methods of analysis will, to a certain degree, be able to counteract any dietary effect, as the dogs could serve as their own controls.

With respect to the sample size, the current study is similar to other feeding trials performing similar analyses (15-18).

In conclusion, our study was unable to demonstrate a significant change in microbiota composition or function in healthy dogs administered with $L k$ at a dose of $3.2 \pm 2.4 \times 10^{7}$ CFU/daily dose for 30 days. Further research will be necessary in order to assess the efficacy of a higher dose or of the combination of $L k$ with other potential probiotics.

\section{DATA AVAILABILITY STATEMENT}

The microbiota datasets generated for this study can be found in the Sequencing reads were deposited in the National Center for Biotechnology Information Sequence Read Archive (NCBI SRA; BioProject ID PRJNA 592436).

\section{ETHICS STATEMENT}

The trial was authorized by the Animal Welfare Committee of the University of Bologna (Protocol No. 3885 of 21/07/2017). Written informed consent was obtained from the owners for the participation of their animals in this study.

\section{AUTHOR CONTRIBUTIONS}

AG and MP were responsible for the conception of the study and data interpretation and wrote the manuscript. AZ and MS performed fecal IgA determination. MF reviewed the manuscript and provided critical suggestions and comments. ST, MB, and $\mathrm{PB}$ analyzed fecal microbiota. RZ performed quality control analysis. All authors discussed the results and approved the final manuscript. 


\section{REFERENCES}

1. Lee WJ, Hase K. Gut microbiota-generated metabolites in animal health and disease. Nat Chem Biol. (2014) 10:416-24. doi: 10.1038/nchembio.1535

2. Grześkowiak $€$, Endo A, Beasley S, Salminen S. Microbiota and probiotics in canine and feline welfare. Anaerobe. (2015) 34:14-23. doi: 10.1016/j.anaerobe.2015.04.002

3. Redfern A, Suchodolski J, Jergens A. Role of the gastrointestinal microbiota in small animal health and disease. Vet Rec. (2017) 181:370. doi: 10.1136/vr.103826

4. Littler RM, Batt RM, Lloyd DH. Total and relative deficiency of gut mucosal IgA in German shepherd dogs demonstrated by faecal analysis. Vet Rec. (2006) 158:334-41. doi: 10.1136/vr.158.10.334

5. Fagarasan S. Evolution, development, mechanism and function of IgA in the gut. Curr Opin Immunol. (2008) 20:170-77. doi: 10.1016/j.coi.2008. 04.002

6. Okanishi H, Sano T, Yamaya Y, Kagawa Y, Watari T. The characteristics of short- and long-term surviving Shiba dogs with chronic enteropathies and the risk factors for poor outcome. Acta Vet Scand. (2013) 55:32. doi: 10.1186/1751-0147-55-32

7. Dandrieux JRS. Inflammatory bowel disease versus chronic enteropathy in dogs: are they one and the same? J Small Anim Pract. (2016) 57:589-99. doi: $10.1111 /$ jsap. 12588

8. Menozzi A, Dall'Aglio M, Quintavalla F, Dallavalle L, Meucci V, Bertini S. Rifaximin is an effective alternative to metronidazole for the treatment of chronic enteropathy in dogs: a randomised trial. BMC Vet Res. (2016) 12:217. doi: 10.1186/s12917-016-0851-0

9. Makielski K, Cullen J, O'Connor A, Jergens AE. Narrative review of therapies for chronic enteropathies in dogs and cats. J Vet Intern Med. (2019) 33:11-22. doi: 10.1111 /jvim. 15345

10. Westermarck E, Skrzypczak T, Harmoinen J, Steiner JM, Ruaux CG, Williams DA, et al. Tylosin-responsive chronic diarrhea in dogs. J Vet Intern Med. (2005) 19:177-86. doi: 10.1892/0891-6640(2005)19<177:tcdid>2.0.co;2

11. Igarashi $\mathrm{H}$, Maeda S, Ohno K, Horigome A, Odamaki T, Tsujimoto H. Effect of oral administration of metronidazole or prednisolone on fecal microbiota in dogs. PLoS ONE. (2014) 9:e107909. doi: 10.1371/journal.pone.0 107909

12. Léger A, Stärk KDC, Rushton J, Nielsen LR. A one health evaluation of the University of Copenhagen Research Centre for control of antibiotic resistance. Front Vet Sci. (2018) 5:194. doi: 10.3389/fvets.2018.00194

13. Schmidt VM, Pinchbeck G, McIntyre KM, Nuttall T, McEwan N, Dawson $\mathrm{S}$, et al. Routine antibiotic therapy in dogs increases the detection of antimicrobial-resistant faecal Escherichia coli. J Antimicrob Chemother. (2018) 73:3305-16. doi: 10.1093/jac/dky352

14. Watkins RR, Bonomo RA. Overview: global and local impact of antibiotic resistance. Infect Dis Clin North Am. (2016) 30:313-22. doi: 10.1016/j.idc.2016.02.001

15. Baillon ML, Marshall-Jones ZV, Butterwick RF. Effects of probiotic Lactobacillus acidophilus strain DSM13241 in healthy adult dogs. Am J Vet Res. (2004) 65:338-43. doi: 10.2460/ajvr.2004.65.338

16. Biagi G, Cipollini I, Pompei A, Zaghini G, Matteuzzi D. Effect of a Lactobacillus animalis strain on composition and metabolism of the intestinal microflora in adult dogs. Vet Microbiol. (2007) 124:160-65. doi: 10.1016/j.vetmic.2007.03.013

17. Pascher M, Hellweg P, Khol-Parisini A, Zentek J. Effects of a probiotic Lactobacillus acidophilus strain on feed tolerance in dogs with nonspecific dietary sensitivity. Arch Anim Nutr. (2008) 62:107-16. doi: 10.1080/17450390801892583

18. Strompfová V, Lauková A, Gancarčíková S. Effectivity of freeze-dried form of Lactobacillus fermentum AD1-CCM7421 in dogs. Folia Microbiol. (2012) 57:347-50. doi: 10.1007/s12223-012-0139-0

19. Gómez-Gallego C, Junnila J, Männikkö S, Hämeenoja P, Valtonen E, Salminen $\mathrm{S}$, et al. A canine-specific probiotic product in treating acute or intermittent diarrhea in dogs: A double-blind placebo-controlled efficacy study. Vet Microbiol. (2016) 197:122-28. doi: 10.1016/j.vetmic.2016.11.015

20. Suchodolski JS. Companion animals symposium: microbes and gastrointestinal health of dogs and cats. J Anim Sci. (2011) 89:1520-30. doi: $10.2527 /$ jas.2010-3377
21. Kim DH, Jeong D, Kang IB, Lim HW, Cho Y, Seo KH. Modulation of the intestinal microbiota of dogs by kefir as a functional dairy product. J Dairy Sci. (2019) 102:3903-11. doi: 10.3168/jds.2018-15639

22. Korsak N, Taminiau B, Leclercq M, Nezer C, Crevecoeur S, Ferauche C, et al. Evaluation of the microbiota of kefir samples using metagenetic analysis targeting the $16 \mathrm{~S}$ and $26 \mathrm{~S}$ ribosomal DNA fragments. J Dairy Sci. (2015) 98:3684-89. doi: 10.3168/jds.2014-9065

23. Pogačić T, Mancini A, Santarelli M, Bottari B, Lazzi C, Neviani E, et al. Diversity and dynamic of lactic acid bacteria strains during aging of a long ripened hard cheese produced from raw milk and undefined natural starter. Food Microbiol. (2013) 36:207-15. doi: 10.1016/j.fm.2013.05.009

24. Carasi P, Díaz M, Racedo SM, De Antoni G, Urdaci MC, Serradell Mde L. Safety characterization and antimicrobial properties of kefirisolated Lactobacillus kefiri. Biomed Res Int. (2014) 2014:208974. doi: 10.1155/2014/208974

25. Carasi P, Racedo SM, Jacquot C, Romanin DE, Serradell MA, Urdaci MC. Impact of kefir derived Lactobacillus kefiri on the mucosal immune response and gut microbiota. J Immunol Res. (2015) 2015:361604. doi: $10.1155 / 2015 / 361604$

26. Toscano M, De Grandi R, Miniello VL, Mattina R, Drago L. Ability of Lactobacillus kefiri LKF01 (DSM32079) to colonize the intestinal environment and modify the gut microbiota composition of healthy individuals. Dig Liver Dis. (2017) 49:261-67. doi: 10.1016/j.dld.2016.11.011

27. Yu Z, Morrison M. Improved extraction of PCR-quality community DNA from digesta and fecal samples. Biotechniques. (2004) 36:808-12. doi: 10.2144/04365ST04

28. Barone M, Turroni S, Rampelli S, Soverini M, D’Amico F, Biagi E, et al. Gut microbiome response to a modern Paleolithic diet in a Western lifestyle context. PLoS ONE. (2019) 14:e0220619. doi: 10.1371/journal.pone.0220619

29. Grellet A, Heilmann RM, Polack B, Feugier A, Boucraut-Baralon C, Grandjean $\mathrm{D}$, et al. Influence of breed size, age, fecal quality, and enteropathogen shedding on fecal calprotectin and immunoglobulin A concentrations in puppies during the weaning period. J Vet Intern Med. (2016) 30:1056-64. doi: 10.1111 /jvim. 14255

30. Masella AP, Bartram AK, Truszkowski JM, Brown DG, Neufeld JD. PANDAseq: paired-end assembler for illumina sequences. BMC Bioinformatics. (2012) 13:31. doi: 10.1186/1471-2105-13-31

31. Caporaso JG, Kuczynski J, Stombaugh J, Bittinger K, Bushman FD, Costello EK, et al. QIIME allows analysis of high-throughput community sequencing data. Nat Methods. (2010) 7:335-36. doi: 10.1038/nmeth.f.303

32. Edgar RC. Search and clustering orders of magnitude faster than BLAST. Bioinformatics. (2010) 26:2460-61. doi: 10.1093/bioinformatics/ btq461

33. Haas BJ, Gevers D, Earl AM, Feldgarden M, Ward DV, Giannoukos G, et al. Chimeric 16S rRNA sequence formation and detection in Sanger and 454-pyrosequenced PCR amplicons. Genome Res. (2011) 21:494-04. doi: $10.1101 /$ gr. 112730.110

34. Altschul SF, Gish W, Miller W, Myers EW, Lipman DJ. Basic local alignment search tool. J Mol Biol. (1990) 215:403-10. doi: 10.1016/S0022-2836(05)80360-2

35. Sandri M, Dal Monego S, Conte G, Sgorlon S, Stefanon B. Raw meat based diet influences faecal microbiome and end products of fermentation in healthy dogs. BMC Vet Res. (2017) 13:65. doi: 10.1186/s12917-017-0981-z

36. Omatsu T, Omura M, Katayama Y, Kimura T, Okumura M, Okumura A, et al. Molecular diversity of the faecal microbiota of Toy Poodles in Japan. J Vet Med Sci. (2018) 80:749-54. doi: 10.1292/jvms.17-0582

37. Tizard IR, Jones SW. The microbiota regulates immunity and immunologic diseases in dogs and cats. Vet Clin North Am Small Anim Pract. (2018) 48:307-22. doi: 10.1016/j.cvsm.2017.10.008

38. Fenimore A, Martin L, Lappin MR. Evaluation of metronidazole with and without Enterococcus faecium SF68 in shelter dogs with diarrhea. Top Companion Anim Med. (2017) 32:100-03. doi: 10.1053/j.tcam.2017.11.001

39. Strompfová V, Kubašová I, Lauková A. Health benefits observed after probiotic Lactobacillus fermentum CCM 7421 application in dogs. Appl Microbiol Biotechnol. (2017) 101:6309-19. doi: 10.1007/s00253-017-8425-z

40. D'Angelo S, Fracassi F, Bresciani F, Galuppi R, Diana A, Linta N, et al. Effect of Saccharomyces boulardii in dogs with chronic enteropathies: double-blinded, placebo-controlled study. Vet Rec. (2018) 182:258. doi: 10.1136/vr.104241 
41. Prado MR, Blandón LM, Vandenberghe LPS, Rodrigues C, Castro GR, Thomaz-Soccol V, et al. Milk kefir: composition, microbial cultures, biological activities, and related products. Front Microbiol. (2015) 30; 6:1177. doi: 10.3389/fmicb.2015.01177

42. Sanders ME. Impact of probiotics on colonizing microbiota of the gut. J Clin Gastroenterol. (2011) 45:S115-S119. doi: 10.1097/MCG.0b013e31822 $7414 \mathrm{a}$

43. Khalesi S, Bellissimo N, Vandelanotte C, Williams S, Stanley D, Irwin C. A review of probiotic supplementation in healthy adults: helpful or hype? Eur J Clin Nutr. (2019) 73:24-37. doi: 10.1038/s41430-0180135-9

44. Delucchi L, Fraga M, Perelmuter K, Cella CD, Zunino P. Effect of native Lactobacillus murinus LbP2 administration on total fecal IgA in healthy dogs. Can J Vet Res. (2014) 78:153-55.

45. Hamilton-Miller JMT, Shah S. Deficiencies in microbiological quality and labelling of probiotic supplements. Int J Food Microbiol. (2002) 72:175-76. doi: 10.1016/s0168-1605(01)00703-6

46. Drago L, De Vecchi E, Nicola L, Colombo A, Gismondo MR. Microbiological evaluation of commercial probiotic products available in Italy. J Chemother. (2004) 16:463-67. doi: 10.1179/joc.2004.16.5.436
47. Weese JS, Martin H. Assessment of commercial probiotic bacterial contents and label accuracy. Can Vet J. (2011) 52:43-6.

48. Bertazzoni Minelli E, Benini A. Relationship between number of bacteria and their probiotic effects. Microb Ecol Health Dis. (2008) 20:180-83. doi: 10.1179/1973947813Y.0000000078

49. Drago L, De Grandi R, De Vecchi E, Toscano M. Antibiotic susceptibility profile of a new Lactobacillus kefiri strain. J Glob Antimicrob Resist. (2016) 4:74-5. doi: 10.1016/j.jgar.2015.10.003

Conflict of Interest: The authors declare that the research was conducted in the absence of any commercial or financial relationships that could be construed as a potential conflict of interest.

Copyright (c) 2020 Gaspardo, Zannoni, Turroni, Barone, Sabetti, Zanoni, Forni, Brigidi and Pietra. This is an open-access article distributed under the terms of the Creative Commons Attribution License (CC BY). The use, distribution or reproduction in other forums is permitted, provided the original author(s) and the copyright owner(s) are credited and that the original publication in this journal is cited, in accordance with accepted academic practice. No use, distribution or reproduction is permitted which does not comply with these terms. 